\title{
CHARACTERIZATION OF DIESEL SPRAY IMAGES USING THE LOGARITHMIC IMAGE PROCESSING FRAMEWORK
}

\author{
CeCILE PETIT ${ }^{1,2}$, Michel JOURLiN ${ }^{1}$ AND WOLFGANG RECKERS ${ }^{2}$ \\ ${ }^{1}$ TSI Laboratory, Traitement du Signal et Instrumentation, UMR 5516 CNRS, Saint-Etienne, France; ${ }^{2}$ Delphi, Technical \\ Center Luxembourg \\ e-mail: petit.cecile@yahoo.com; wolfgang.reckers@delphi.com; michel.jourlin@univ-st-etienne.fr \\ (Accepted September 12, 2007)
}

\begin{abstract}
The increasing levels of emission standards in Diesel Engines require a detailed understanding, combustion and after treatment. This paper focuses on the spray development as one key parameter in the mixture preparation. The presentation of a methodology to differentiate the internal symmetry of spray images taken under different environmental conditions is presented. In a first step, a preprocessing is performed, then an image re-centering is made using the logarithmic average, afterwards different symmetry axes based on grey levels or on the plume boundary are calculated and, finally, the logarithmic distance characterizing the spray plume internal symmetry is computed. This distance gives more importance to the high grey level pixels, so using our optical setup, it characterizes the liquid continuous core symmetry. The methodology relies on the logarithmic image processing framework, providing a set of specific algebraic and functional operations to analyze images. This paper is an application of the logarithmic image processing framework on Diesel spray characterization. This is a step further in the quantitative diesel spray characterization by means of image analysis. The presented method can be applied to Diesel sprays illuminated with polychromatic or monochromatic light, under atmospheric or pressurized conditions.
\end{abstract}

Keywords: diesel spray characterization, grey level symmetry axes, internal symmetry, logarithmic image processing.

\section{INTRODUCTION}

In Diesel engines, the stricter levels of emission standards require an increasing understanding of combustion process. A key element in combustion is the spray injected into the combustion chamber. Different approaches, coupling (or not) different hydraulic and mechanical characterizations, internal flow pattern and optical spray diagnostics exist to investigate on the spray.

To control the pollutant emissions, the spray length and shape have to be adapted to the combustion chamber. If the spray length is high, it could impinge on the spray chamber wall, increasing emission of soot and unburned hydrocarbons. The spray inside the combustion chamber should be well distributed, neither too rich nor too poor in order to optimize the combustion. Controlling the spray symmetry would enable adapting the injector to the combustion chamber, optimizing the combustion and the produced energy, reducing the pollutant emissions.

In Soteriou (2000) are presented different large scale models which are analyzed on a visualization rig and on a laser stand, to understand how the spray shape and the internal flow are affected by pressure, degree of opening, entry and exit orifice. Payri (2005) focuses on the influence of cavitation on the spray behavior by means of CFD, momentum and velocity studies. Kull and Krüger (2004) couple flow, spray momentum and macroscopic visualization to investigate on spray uniformity.

Other studies present non intrusive spray visualization and image processing techniques to analyze the spray uniformity. Feature extraction algorithms, based on likelihood ratio test (Pastor et al., 2001) or on the histogram analysis (Cronhjort and Wahlin, 2004) are convenient to get the spray shape and the macroscopic parameters. Delacourt et al. (2005) study the injection pressure influence on spray macroscopic parameters as penetration, angle, area and volume. Petit et al. (2005) present a methodology to calculate the virtual spray origin (virtual injection center), the single plume shape inertia axes, and tools to study the single plumes internal and external symmetry.

A short overview about the experimental setup and the image acquisition is presented first. Preprocessing and spray origin are introduced as a base for the single 
plume selection and evaluation. Then different methods for spray symmetry calculation are discussed. After an introduction of the Logarithmic Image Processing (LIP) basics, the internal single spray plume characterization is presented in detail. Finally the methods are applied to spray shapes and the results are presented.

\section{MATERIALS \& METHODS}

\section{EXPERIMENTAL SETUP AND IMAGE ACQUISITION}

The multi-hole injector is mounted in an atmospheric test stand or in a pressure chamber where the air pressure and temperature can be set. The high pressure pump supplies pressurized (between $20 \mathrm{MPa}$ and $250 \mathrm{MPa}$ ) test fluid (ISO 4113) to the injector. The images were acquired at $700 \mu$ s after start of injection. The optical setup is presented in Fig. 1.

The system acquires spray images synchronized with the injector actuation. Multiple pictures are recorded at different delays after the start of injection.

The light source (stroboscopic white light or NdYAG) illuminates the whole spray. The scattered light is recorded by the CCD camera (LaVision, single shot or high speed, 12 bits). In order to minimize light from the background, a black screen is applied behind the injector tip.

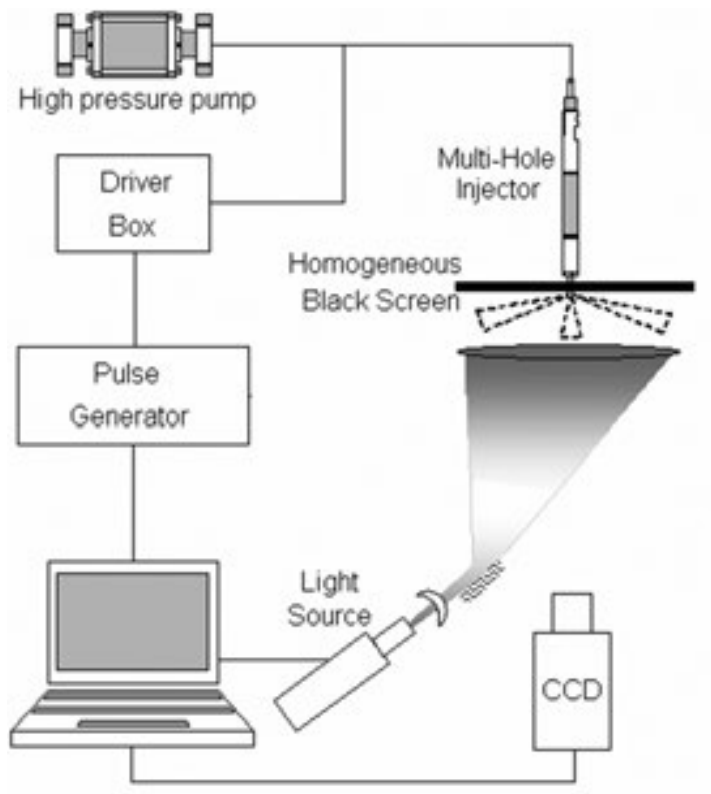

Fig. 1. The optical device used for image acquisition.

\section{PREPROCESSING, VIRTUAL SPRAY ORIGIN CALCULATION AND SINGLE PLUMES SELECTION}

It (Fig. 1) provides a homogeneous background on the images but preprocessing is still necessary: a background image (without injection) can be subtracted from the raw image, and a reference image (taken with a white screen) can be used to correct the light non homogeneity.

Additional filtering may be necessary; median filter can be used carefully as the contrast is sensitive to filtering.

From these images first the virtual spray origin (VSO) is calculated, then single plumes are selected and, finally, the symmetry axis and parameter computed.

The VSO is a very important point as it is a base for the single spray plumes characterization: it can be selected as the origin for the penetration and angle. These parameters are extremely sensitive to the VSO position, a slight shift of the VSO changes the calculated penetration and angle significantly.

The virtual spray origin (VSO) calculation was first presented in Petit et al. (2005). A raw virtual spray origin is calculated (RVSO), then an accurate virtual spray origin is deduced from a Voronoi diagram (AVSO). To get the RVSO, the principal axes of the single plumes boundaries are calculated, as the first principal axes meet in the VSO vicinity, the AVSO is the barycenter of these axes intersections. Then, the nearest points to the AVSO belonging to the spray are computed, they are the basis of the Voronoi diagram. The Voronoi vertex barycenter is the AVSO.

As the grey levels of the jet will be used for the main part of the calculation, the need of this process is to get the best single plume image, taking into account all pixels belonging to the plume.

The initial preprocessed image and the AVSO are used to get the single plume images. A circle profile, centered at the AVSO (see the red circle on Fig. 2b), intersects the individual plumes (Seneschal et al., 2003), which is shown in blue in Fig. 2b. The midangles (dashed green lines, Fig. 2b) between these marks represent the limits between the individual plumes. A single plume image can be derived (Fig. 2c) from two mid-angles.

The next section presents different methodologies to calculate the grey levels symmetry axes. 


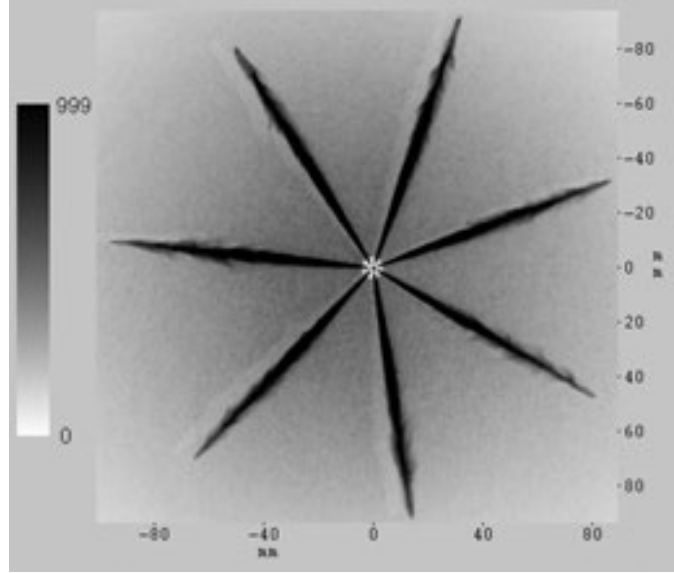

(a)

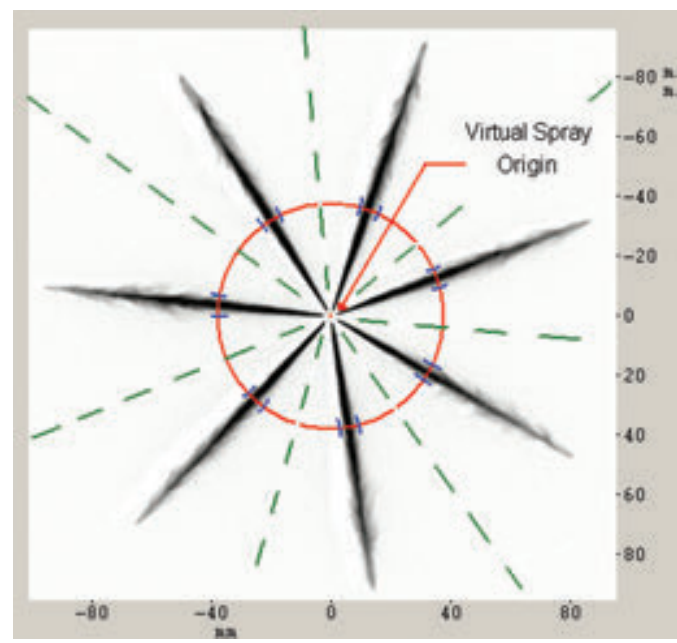

(b)

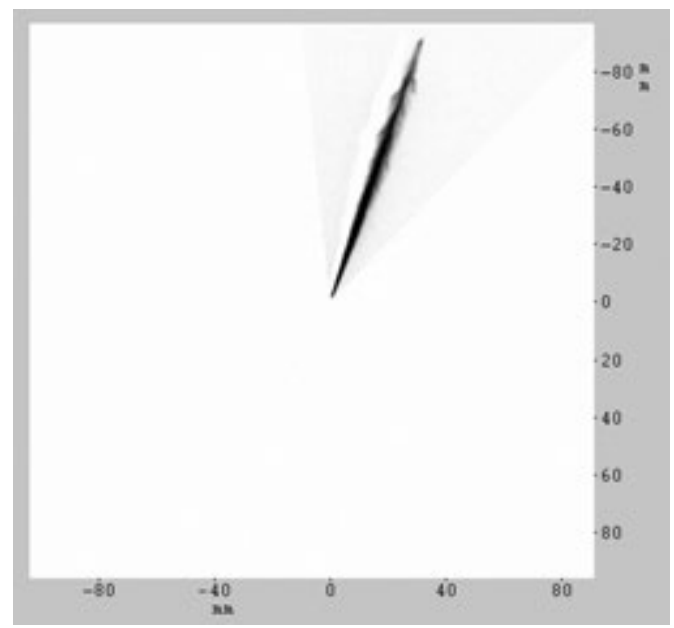

(c)

Fig. 2. Different steps to get the single plume image. a) The spray raw image with the grey level scale bar. b) Preprocessed image with the virtual spray origin, method to get the single plume image presented in (c). Units: 1 pixel $=1 \mathrm{~mm}$.

\section{SINGLE PLUME GREY LEVEL SYMMETRY AXES}

A single spray plume symmetry axis can be calculated from its boundary as presented in Petit et al. (2005). Nevertheless, the quantified image of the spray describes the physical process of the flow evolution. An axis representing the spray development and a parameter showing its symmetry would be a useful tool for spray characterization. The axis calculated with the boundary does not permit this type of analysis but the use of the spray image grey levels enables the calculation of the spray development axis (SDA) describing the flow evolution. In the perfect case, the SDA starts at a virtual point, the AVSO, follows the main flow development all along the plume shape and is centered compared to this one. In the Results and discussion part, it will be shown that, depending on the particular plume shape and on the investigation aim, other axis gives a better description of the flow.

The principle is to calculate the mean square regression line, using the pixel grey levels as weights. Defining a pixel of coordinates $\left(x_{i}, y_{j}\right)$ of grey level $p_{i j}$, the searched line equation of $y=a x+b$ can be found thanks to the mean square method:

$$
\begin{gathered}
a=\frac{\sum_{\left(x_{i}, y_{j}\right) \in \text { plume }} p_{i j} x_{i} y_{j}-N \cdot \bar{x} \cdot \bar{y}}{\sum_{\left(x_{i}, y_{j}\right) \in \text { plume }} p_{i j} x_{i}^{2}-N \cdot \bar{x}^{2}}, \\
b=\bar{y}-a \cdot \bar{x} \\
\bar{x}=\frac{1}{N} \sum_{\left(x_{i}, y_{j}\right) \in \text { plume }} p_{i j} x_{i} \\
\bar{y}=\frac{1}{N} \sum_{\left(x_{i}, y_{j}\right) \in \text { plume }} p_{i j} y_{j} . \\
N=\sum_{\left(x_{i}, y_{j}\right) \in \text { plume }} p_{i j}
\end{gathered}
$$

This defined line is presented in Figs. 5b-i in blue.

As shown in Fig. 5d, the so-defined line might not come from the AVSO. To improve the algorithm, the condition that the symmetry axis passes by the $\operatorname{AVSO}\left(x_{0}, y_{0}\right)$ has been integrated. Concerning the searched equation, the value $b$ is now known, the left parameter is the slope $a$, which can be calculated as the average slope of the lines passing by the AVSO, using the weight $p_{i j}$ (see Figs. $5 c$ and d, in green). 


$$
\begin{array}{r}
a=\frac{\sum_{\left(x_{i}, y_{j}\right) \in \text { plume }} p_{i j} a_{i j}}{\sum_{\left(x_{i}, y_{j}\right) \in \text { plume }} p_{i j}}, \\
b=y_{0}-a \cdot x_{0} \\
a_{i j}=\frac{y_{j}-y_{0}}{x_{i}-x_{0}} .
\end{array}
$$

As shown in Fig. 5b, the green line is not centered at the plume shape center and does not go in the main flow direction. Another factor has to be taken into account: the distance from the AVSO to the current pixel. Two different solutions exist. They depend on the information wanted at the end: the first one gives more importance to the nearest pixels of the AVSO, the second one more importance to the farthest pixels to the AVSO.

The first solution, presented by the red lines in Figs. 5d-i, may be expressed as:

$$
\begin{gathered}
a=\frac{\sum_{\left(x_{i}, y_{j}\right) \in \text { plume }} \frac{p_{i j}}{r_{i j}} a_{i j}}{\sum_{\left(x_{i}, y_{j}\right) \in \text { plume }} \frac{p_{i j}}{r_{i j}}}, \\
b=y_{0}-a \cdot x_{0} \\
r_{i j}=\sqrt{\left(x_{i}-x_{0}\right)^{2}+\left(y_{j}-y_{0}\right)^{2}} .
\end{gathered}
$$

This solution gives an axis following the liquid continuous core flow evolution. If the main flow is to be investigated, the distance constraint must be changed in order to obtain an axis centered in the shape all along the plume length.

The second solution, giving more importance to the farthest pixels of the AVSO and presenting a more symmetric line all along the plume, shown in yellow in Figs. 5b-i, may be calculated using:

$$
\begin{gathered}
a=\frac{\sum_{\left(x_{i}, y_{j}\right) \in \text { plume }} p_{i j} r_{i j} a_{i j}}{\sum_{\left(x_{i}, y_{j}\right) \in \text { plume }} p_{i j} r_{i j}} . \\
b=y_{0}-a \cdot x_{0}
\end{gathered}
$$

This last way of computing the symmetry axis provides the Spray Development Axis (SDA) as this line fits the plume along the flow evolution.

The SDA and the other axes are the base for the internal symmetry characterization after the images referencing using the Logarithmic Image Processing
(LIP) framework (Jourlin and Pinoli, 2001). The topic of the next section is firstly the presentation of the LIP model, secondly the explanation of its advantage in Diesel spray application, thirdly the discussion of the images referencing to a value and, finally, the presentation of the symmetry parameter calculation.

\section{INTERNAL SYMMETRY CHARACTERIZATION}

The LIP (Jourlin and Pinoli, 2001) has been selected as the mathematical framework for this application: in the case of Diesel sprays, the object is dense and is composed of droplets. As shown in Fig. 1 , the front illumination, which can be monochromatic or polychromatic, impacts the spray. The particles composing the light penetrate the medium more or less deeply before being reflected and then captured by the CCD camera. It corresponds to the definition of semi-reflected images. Depending on the current operating and ambient conditions, the spray will be more or less dense; the light will more (monochromatic light) or less (polychromatic light) penetrate the spray (Delacourt et al., 2005) so the image contrast will be affected. That is why a pre-requisite of the internal distance computation will be the image dynamic referencing, so that any image taken with monochromatic or polychromatic light might be compared. Besides this pre-requisite, the grey tone scale limits [0,M, is another one: 0 and $M$ represent the white and the black value, respectively. In other words, 0 and $M$ correspond to the total transparency and total opacity, respectively. It is exactly the case using the setup presented in Fig. 1. The value $M$ is defined by the camera itself: it is a 12 bytes camera, so $\mathrm{M}=212=$ 4096.

The LIP fundamentals are briefly explained, followed by an overview of the operations and specific algebraic.

The LIP theory is a mathematical framework providing a set of specific algebraic and functional operations (addition, subtraction, multiplication, differentiation, integration, convolution...) and structures that are well adapted to the representation and processing of non-linear images and, more generally, of non-linear signals, values in a bounded intensity range. The physical absorption/transmission laws may be expressed within the LIP mathematical framework, it has been also proved that it is pretty close to the human visualization system.

A medium is considered as a sequence of elementary successive slides. Each slide may be considered as a filter that can be identified to its 
associated grey level function (grey tone function, its pixels value). Two different slides, denoted $f$ and $g$, may be superimposed, and the resulting medium, the "sum between $f$ and $g$ " is noted $f \Delta g$, where the symbol $\mathbf{A}$ designates the LIP addition. $f \Delta \mathrm{A}$ is defined by:

$$
f \Delta g=f+g-\frac{f g}{M} .
$$

It has been proved that this LIP addition has several properties:

Associativity:

$$
\begin{gathered}
\forall f, g, h \in \mathrm{I}, \\
(f \otimes g) \otimes h=f \otimes(g \otimes h)
\end{gathered}
$$

Neutral element:

$$
\begin{gathered}
\forall f \in \mathrm{I}, \\
0 \Delta f=f
\end{gathered} .
$$

Commutativity:

$$
\begin{gathered}
\forall f, g \in \mathrm{I}, \\
f \mathbf{\Delta} g=g \boldsymbol{\Delta} f
\end{gathered}
$$

It might be interesting to amplify a grey tone function $f$, by a value $\alpha$ : let us consider that the original image $f$ is not enough contrasted, an idea would be to get the image $\mathrm{f}$ amplified by a value $\alpha$, in order to have the signal given by $\alpha$ slices of $f$, noted $\alpha \Delta f$. This LIP scalar multiplication is defined by:

$$
\begin{gathered}
\forall \alpha \in \mathfrak{R}^{+}, \\
\alpha \Delta f=M-M\left(1-\frac{f}{M}\right)^{\alpha} .
\end{gathered}
$$

The LIP scalar multiplication also satisfies these properties:

Associativity:

$$
\begin{gathered}
\forall \alpha, \beta \in \mathfrak{R}^{+}, \forall f \in \mathrm{I}, \\
\alpha \Delta(\beta \Delta f)=(\alpha \beta) \Delta f
\end{gathered}
$$

Unit element:

$$
\begin{gathered}
\forall f \in \mathrm{I}, \\
1 \Delta f=f
\end{gathered} \text {. }
$$

Double distributivity:

$$
\forall \alpha \in \mathfrak{R}^{+}, \forall f, g \in \mathrm{I}:
$$

$$
\begin{aligned}
\alpha \Delta(f \Delta g) & =(\alpha \Delta f) \Delta(\alpha \Delta g) . \\
\forall \alpha, \beta & \in \mathfrak{R}^{+}, \forall f, g \in \mathrm{I}: \\
(\alpha+\beta) \Delta f & =(\alpha \Delta f) \Delta(\beta \Delta f)
\end{aligned}
$$

Furthermore, it has been proved that the natural order relation, denoted $\geq$, exits in the I space, thus for two grey tone functions $f$ and $g$ such as $f \geq g$, their difference $f \Delta g$ is defined by:

$$
f \Delta g=M \frac{f-g}{M-g} \text {. }
$$

Now that the LIP framework and its use in the case of Diesel sprays and its different operations have been presented, let us show how it can be applied with Diesel spray images.

In order to compare images acquired in different conditions and setups, these images are set to the same reference: the identical logarithmic average, which will make it possible to get logarithmically the same contrast for all spray plumes.

Let us consider the part of the image (non zero) only presenting the Diesel spray. The threshold (noted $t h$ ) between the background and the object can be deduced from an automatic segmentation, as Pastor et al. (2001) or Cronhjort and Wahlin (2004), or the Entropy maximization (Pun, 1981). The spray logarithmic average is defined using the LIP framework; it represents the dynamic grey level average all over the spray. Let us consider a pixel $x$, its grey level is $f(x)$. The logarithmic average is defined by:

$$
\begin{gathered}
\forall f(x) \geq t h, \\
\mu_{\log }(f)=\frac{1}{n_{p}} \Delta\left(\sum_{x \in \text { plume }}^{\Delta} f(x)\right),
\end{gathered}
$$

with $n_{p}$ the number of pixels within the plume, $\sum^{\Delta}$ represents the LIP summation.

Using the properties of the LIP addition and multiplication by a scalar, it can be proved that:

$$
\mu_{\log }(\lambda \Delta f)=\lambda \Delta \mu_{\log }(f) \text {. }
$$

Let us assume different spray plumes are defined with $f_{i}$. As the aim is to set the logarithmic average to a value $\beta$, the equation to be solved is: 


$$
\lambda_{i} \Delta \mu_{\log }\left(f_{i}\right)=\beta \text {. }
$$
to:

Using equation 12, it can be proved to be equivalent

$$
\lambda_{i}=\frac{\ln \left(\frac{M-\beta}{M}\right)}{\ln \left(1-\frac{\mu_{\log }\left(f_{i}\right)}{M}\right)} .
$$

So, to get the spray images of logarithmic average $\beta$, only one operation has to be applied: $\lambda_{i} \Delta f_{i}$, where $\lambda_{i}$ is defined in equation 20 .

In the next step, the symmetry axes (see above) are calculated and then mirrored along themselves. An example is shown in Fig. 3.

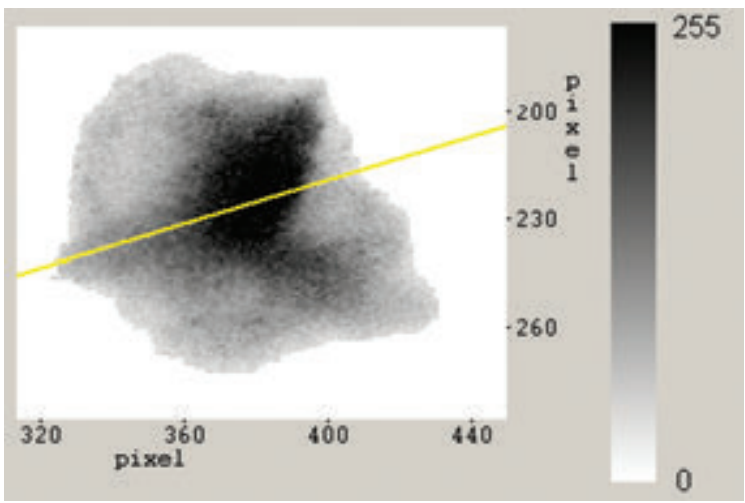

(a)

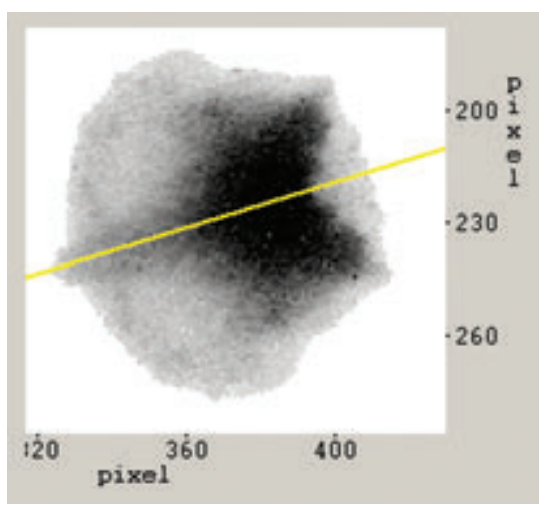

(b)

Fig 3. a) The input spray plume with the grey level scale bar. b) The mirror spray plume about the symmetry axis.

The calculation of the internal symmetry parameter is made comparing the half spray plumes: the identical parts between the images are deleted as shown in the next figure:

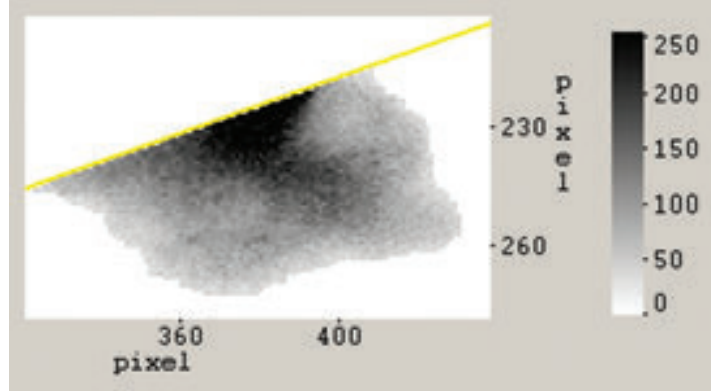

(a)

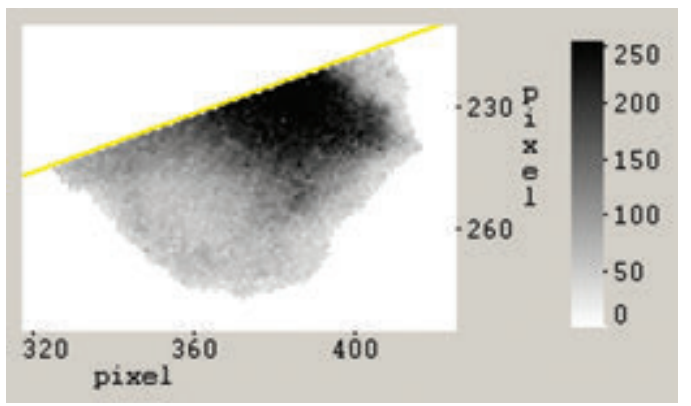

(b)

Fig 4. Images used to calculate the internal symmetry with their grey level scale bars. a) The half input spray plume. b) The half mirror spray plume.

The internal symmetry characterization is based on the comparison between these images applying the LIP.

In order to quantify the internal symmetry, the logarithmic distance $d_{\Delta}\left(f_{h}, g_{h}\right)$ [grey level] is introduced: it is defined as the logarithmic average of the logarithmic contrasts between the half original spray plume $f_{h}$ and the half mirrored spray plume $g_{h}$.

$d_{\Delta}\left(f_{h}, g_{h}\right)=\frac{1}{n} \Delta\left[\sum_{x \in \text { plume }}^{\Delta} C^{\Delta}\left(f_{h}(x), g_{h}(x)\right)\right]$,

with $n$ the number of couples $\left(f_{h}(x), g_{h}(x)\right), x$ the pixel.

The logarithmic contrast $C^{\Delta}\left(f_{h}(x), g_{h}(x)\right)$ between the input image $f_{h}$ and the mirror image $g_{h}$, for the pixel $\mathrm{x}$ may be defined by:

$$
\begin{array}{r}
C^{\Delta}\left(f_{h}(x), g_{h}(x)\right)=\left|f_{h}(x) \Delta g_{h}(x)\right|, \\
C^{\Delta}\left(f_{h}(x), g_{h}(x)\right)=\frac{\left|f_{h}(x)-g_{h}(x)\right|}{1-\frac{\operatorname{Min}\left(f_{h}(x), g_{h}(x)\right)}{M}} .
\end{array}
$$

The advantages of this method over the one presented in Petit et al. (2005) are that images taken 
with different setups (monochromatic, polychromatic light) are be compared, the axis presenting the spray development is calculated and finally the high grey level pixels have more influence on the results and the low ones. It means that using our optical setup, this distance determines the liquid continuous core internal symmetry.

If needed, the level of internal symmetry discrepancies might be enhanced, using a classification of the spray plume pixel grey levels in a fewer number of classes. The use of the k-means algorithm, combined with a reduced number of classes, permits the differences to be enhanced. This algorithm can also be used in the logarithmic way, calculating the logarithmic barycenter of the class and the logarithmic middle between two consecutive logarithmic centers.

\section{RESULTS \& DISCUSSION}

The calculation of different symmetry axes, as well as the internal symmetry logarithmic distance can be applied to different types of spray plumes. Images taken with monochromatic light in the atmospheric chamber, with polychromatic light under environmental or pressurized environment will be analyzed.

The results for one single plume are first presented and described, and then the results for the other plumes are compared. The images are presented in a large scale in Fig. 5.

Table 1. The logarithmic distances for different images and different axes.

\begin{tabular}{|c|c|c|c|c|c|c|}
\hline Axis color & & & & & & \\
\hline $\begin{array}{c}\text { log dist } \\
\text { [grey level] }\end{array}$ & $\begin{array}{c}\text { Boundary } \\
\text { Axis }\end{array}$ & $\begin{array}{c}\text { Boundary } \\
\text { AVSO } \\
\text { Axis }\end{array}$ & $\begin{array}{c}\text { Grey Levels } \\
\text { Axis }\end{array}$ & $\begin{array}{c}\text { Grey Levels } \\
\text { AVSO Axis }\end{array}$ & $\begin{array}{c}\text { Grey Levels } \\
\text { AVSO 1/r } \\
\text { Axis }\end{array}$ & $\begin{array}{c}\text { Grey Levels } \\
\text { AVSO r } \\
\text { Axis }\end{array}$ \\
\hline b & 32.1262 & 29.8248 & 42.5043 & 39.2772 & 44.216 & 36.6905 \\
\hline c & 57.9841 & 60.7297 & 54.0499 & 51.9095 & 46.0096 & 53.9657 \\
\hline d & 34.0133 & 40.0636 & 41.9648 & 37.154 & 38.9882 & 36.7521 \\
\hline e & 25.5033 & 40.0424 & 38.6211 & 43.2887 & 50.1469 & 41.1883 \\
\hline f & 51.3531 & 45.9514 & 51.1761 & 41.5764 & 45.712 & 40.5719 \\
\hline g & 70.2829 & 57.9101 & 53.9168 & 61.7735 & 67.6969 & 53.3038 \\
\hline h & 42.6478 & 36.5449 & 43.8505 & 34.4418 & 38.5557 & 33.5251 \\
\hline i & 29.5053 & 24.8508 & 38.5879 & 24.3915 & 24.9386 & 24.2165 \\
\hline $\begin{array}{c}\text { sum of } \\
\text { distances }\end{array}$ & 317.9127 & 295.8753 & 326.0503 & 290.5239 & 306.117 & 279.0256 \\
\hline
\end{tabular}

The references $\mathrm{b}$ to $\mathrm{i}$ of column 1 correspond to the spray plumes presented in Fig. 5. The columns 2 to 7 represent the axes presented previously. The columns colours correspond to the axis colours of Fig. 5. For each axis, and image the logarithmic distance is calculated and presented in table 1 . The images logarithmic distances are summated for each axis. This summation has no physical significance but it represents the different methodologies result taking into account the complete panel of sprays. It enables the comparison of the methodologies.

Each axis and logarithmic distance was tested on his own: all methodologies have strengths and weaknesses. Our aim is to do a balanced judgment testing a panel of spray plumes shapes acquired in different test conditions.

The plume of Fig. 5b. is used to describe the different types of axis and the resulted internal symmetry. The light blue axis has been calculated using the boundary, the calculation of the logarithmic distance using this axis gives the rough grey level distribution inside the plume. This distance is quite small which indicates good grey level distribution homogeneity. The pink axis has also been calculated using the plume boundary but now, it is forced to pass by the VSO. This time, the distance is slightly smaller, even if the axis position has only one pixel of difference with the previous axis. As the high grey level pixels have more influence on the logarithmic distance and as the pink axis goes towards the high grey level center (see upper part of the plume), the distance is similar but slightly smaller than the one with the light blue axis. If the aim of the researcher is to identify the rough grey level distribution inside the plume, he should use this axis, as it goes in the direction of the VSO and as it is centered compared to the shape.

The aim of the study using the SDA is to know the plume internal symmetry comparing to the flow development. In this case, the grey levels must be taken into account for the axis computation. It is the case for the other axes. The blue axis is the rough symmetry axis using the grey levels. This time, the axis is less centered compared to the shape than the two previous ones. It explains why the distance is bigger. Adding the constraint that it passes through the VSO gives a better result (green axis which is more centered at the upper part) even if it is not centered at the end of the plume. Looking at the result for the red axis, which passes by the VSO and gives more importance to the pixels nearer to this point, shows that the distance is high: even if the axis is centered at the upper part of the plume, it is not at all at the lower part. Note also that it is the worse symmetry axis all along the plume compared to the other ones and the distance is higher, which is logical. Nevertheless, this type of axis corresponds to the liquid continuous core axis, as more importance is given to the nearest pixels to the VSO. Finally, the last axis (yellow) passes through the VSO and gives more importance to the farthest pixels to the VSO is 


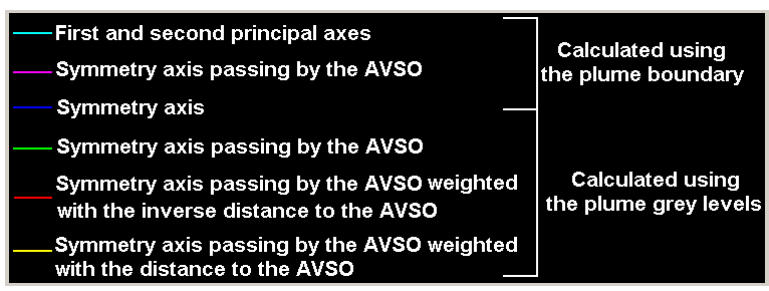

(a)

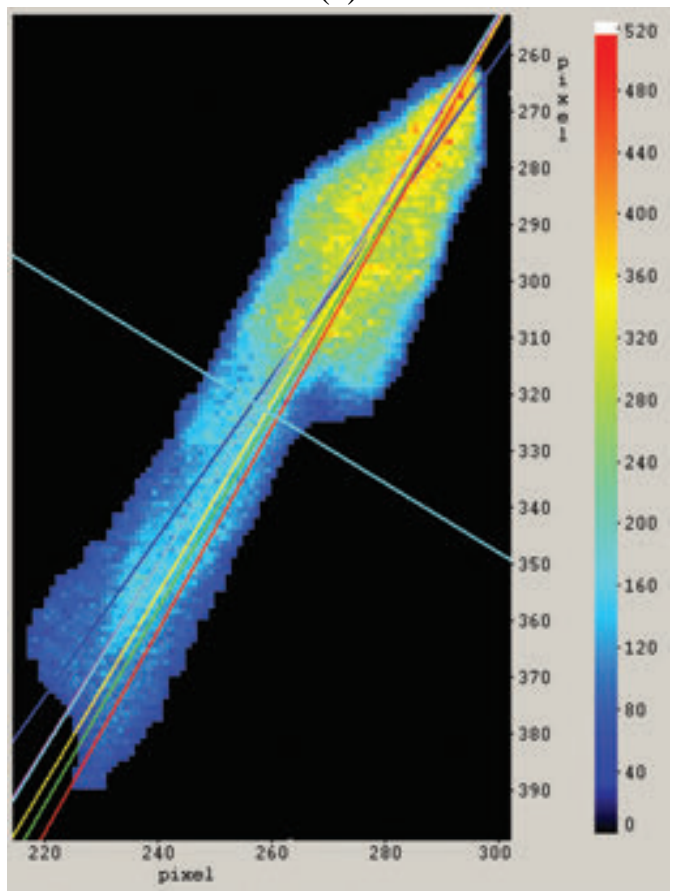

(b)

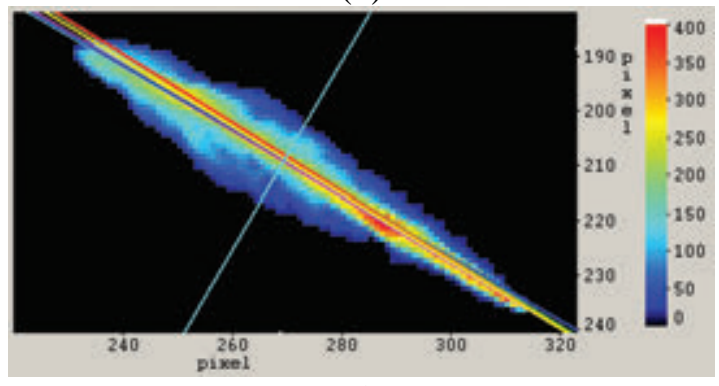

(f)

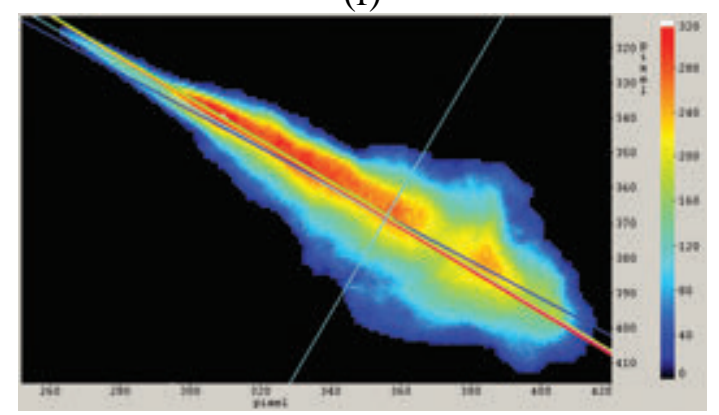

(h)

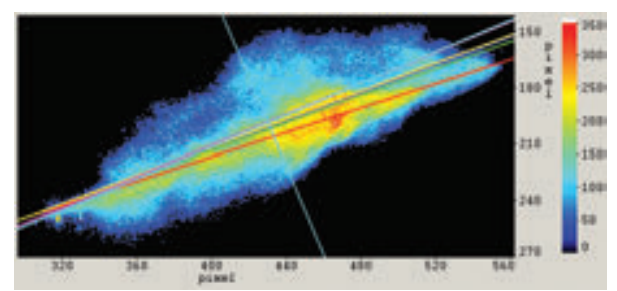

(c)

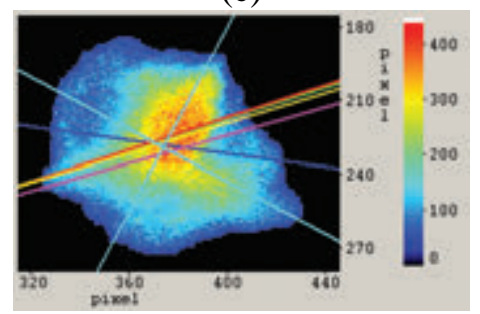

(d)

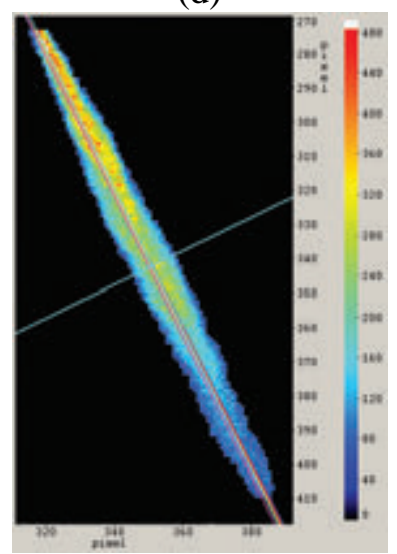

(e)

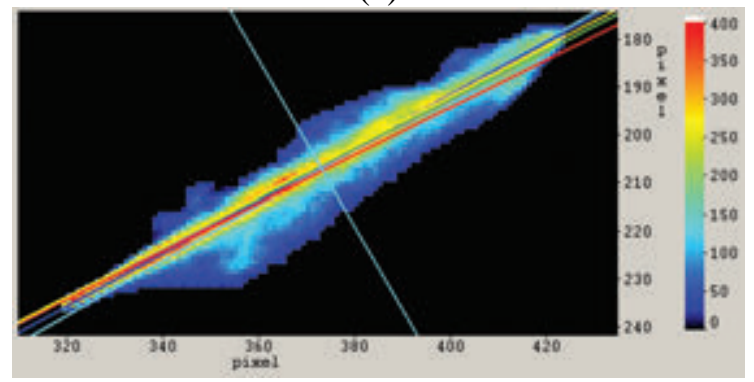

(g)

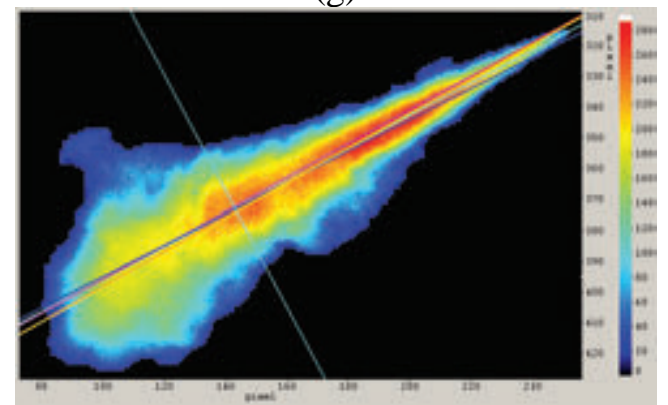

(i)

Fig. 5. Axes representation a) and analyzed images. b), c), d) and e) taken with monochromatic light (ND-YAG) under environmental conditions. f), g) taken with polychromatic light, under atmospheric conditions. h), i) taken with polychromatic light under pressurized conditions (25 Bar environmental pressure, $200 \mathrm{C}$ environmental temperature). 
more centered all along the plume compared to the previous one. This time, the distance is lower. Note that it is the smallest distance with the axes calculated using the grey levels: this axis is the SDA as it shows the flow symmetry all along the plume. Another proof is the look at the distances summation for all images $5 \mathrm{~b}$ to $5 \mathrm{i}$. The one calculated with the yellow axis is the lowest which characterizes the internal symmetry of the flow development.

Thanks to the images re-centering, the different images, taken under different environmental conditions and with different light sources, can be compared.

Figs. 5c and 5g present highly asymmetric plumes. The distances are high, whatever axis is used: It proves, as seen on the images, that one half part is completely different compared to the other. For plume $5 \mathrm{c}$, which is wide, one half part contains more high grey level pixels compared to the other. For plume $5 \mathrm{~g}$, which is thin, one half part is thin and wavy compared to the second one.

The plume $5 \mathrm{~d}$ is wide but it is more symmetric than plumes $5 \mathrm{c}$ and $5 \mathrm{~g}$. Its shape is regular (circle) even if it is not expected for a Diesel spray. Looking at the axis shows that some present less symmetry but the SDA symmetry is better. The distance of plume $5 \mathrm{~b}$ is similar as their internal symmetry are not perfect, specifically taking about the high grey levels: for plume $5 b$, there are high grey level differences (see the "dots" distribution) which are not equal in the two half parts. In plume $5 \mathrm{~d}$, the high grey levels are not very symmetric one half part to the other.

Fig. 5e presents a very thin plume that seems pretty symmetric (see the light blue axis, the rough grey level distribution). Looking at the results for the SDA, one observes a long distance, which can be explained by the plume shape: as it is very thin, one pixel symmetric point can easily fall into the background, making the distance higher. So, for thin plumes the use of the axes calculated using only the boundary (light blue axis) seems appropriate.

Fig. $5 f$ presents a plume with a regular shape but with waves inside: the grey level distribution are not symmetric compared to the axes (see yellow, red, light green and light blue pixels), which gives a high logarithmic distance.

Looking at plume $5 \mathrm{~h}$, which is wide, shows less waves inside the plume compared to $5 \mathrm{f}$ - but they still exist. It causes a better symmetry.
Finally, plume 5i, which is wide, presents very homogeneous and centered axes causing a low distance. The internal plume symmetry is the best; a look at the other plumes proves that it is the best.

\section{CONCLUSION}

A method was presented to re-center images taken under different circumstances in order to compare them. Several symmetry axes based on the grey levels have been presented and the calculation of the spray development axis has been introduced.

A parameter representing the internal symmetry of a spray plume, based on the logarithmic image processing model, which gives more importance to high grey level pixels, has been presented. It has been shown that these methods permit a comparison between images taken under different environmental conditions and those with different light sources (monochromatic, polychromatic). This method can be applied to wide plumes but is limited for thin ones. In this case, the calculation with the boundary axes (light blue axis) is required.

The calculation of the logarithmic distance for different symmetry axis shows weaknesses and strengths for all methods. The axis choice should be made depending on the plume shape and on the investigation aim: a rough internal symmetry study can be made using the axis calculated using the boundary and passing by the VSO (pink axis). If the research aim is to study the liquid continuous core, the axis calculated using the grey levels, passing by the VSO and giving more importance to the nearest pixels to the VSO should be used (red axis). In the case of the main flow development study, the SDA would be required (yellow axis). Finally, for specific spray plume shapes, as for very thin ones, the raw axis calculated using the boundary (light blue axis) would be recommended.

Studying the spray symmetry could be done using the SDA and the best spray plume regarding this methodology is referenced as i in Fig. 5.

The next step for the spray main flow investigation can be made in different ways. A solution would be to compare the SDA with the one of the drawings and to compare their symmetry parameters. Another solution would be to compare the SDA with the end of injection axis and to observe their symmetry parameters. 


\section{NOMENCLATURE}

\section{Roman:}

a: $\quad$ Line slope.

$a_{i j}$ : $\quad$ Line slope passing by the AVSO and by $\left(x_{i}, y_{j}\right)$.

$b$ : Line origin coefficient.

$C^{\Delta}$ : Logarithmic contrast between two pixels.

$d_{\Delta}$ : Logarithmic distance between two images.

$f: \quad$ Image.

$f_{h}$ : Half original spray plume image.

$f_{i}$ : $\quad$ Spray plume image.

g: Image.

$g_{h}: \quad$ Half mirrored spray plume image.

$h$ : Image.

I: $\quad$ Positive vector cone.

M: $\quad$ Image maximal grey level value, never attained. For grey levels coded on 8 Bits, $M$ is equal to 256.

$n: \quad$ Number of couples $\left(f_{h}(x), g_{h}(x)\right)$ taken into account in the logarithmic distance calculation.

$n_{p}$ : $\quad$ Number of pixels within the spray plume.

$N$ : $\quad$ Pixels belonging to the plume grey level sum.

$p_{i j}$ : $\quad$ Pixel $\left(x_{i}, y_{j}\right)$ grey level.

$r_{i j}$ : Distance from the pixel $\left(x_{i}, y_{j}\right)$ to the AVSO.

th: Single spray plume image segmentation threshold.

$x: \quad$ One pixel.

$\bar{x}$ : Weighted $\mathrm{x}$ coordinates average of the pixels belonging to the plume.

$\left(x_{0}, y_{0}\right)$ : AVSO coordinates.

$\left(x_{i}, y_{j}\right)$ : One pixel coordinates.

$\bar{y}$ : $\quad$ Weighted y coordinates average of the pixels belonging to the plume.

\section{Greek:}

$\alpha$ : $\quad$ Real positive number.

$\beta$ : $\quad$ Real positive number.

$\lambda$ : $\quad$ Real positive number.

$\lambda_{i}$ : $\quad$ Search real number to refer the spray plume $f_{i}$ logarithmic average. $\mu_{\text {log }}$ : Spray plume logarithmic average.

\section{Mathematics symbols:}

A : Logarithmic addition.

$\Delta: \quad$ Logarithmic multiplication.

$A$ : Logarithmic subtraction.

$\sum^{\Delta}$ : Logarithmic summation.

Acronyms:

AVSO:Accurate Virtual Spray Origin.

CCD: Charged Coupled Device.

CFD: Computer Fluid Dynamics.

LIP: Logarithmic Image Processing.

RVSO: Raw Virtual Spray Origin.

SDA: Spray Development Axis.

VSO: Virtual Spray Origin

\section{REFERENCES}

Cronhjort A, Wahlin F (2004). Segmentation algorithm for diesel spray image analysis. Applied Optics 43(32): 5971-80.

Delacourt E, Desmet B, Besson B (2005). Characterisation of very high pressure diesel sprays using digital imaging techniques. Fuel 84:859-67.

Jourlin M, Pinoli JC (2001). Logarithmic image processing, the mathematical and physical framework for the representation and processing of transmitted images. Adv Imag Elect Phys 115:129-94.

Kull E, Krüger G (2004). Correlation of spray symmetry with mass and momentum distribution of multihole diesel nozzles. THIESEL proceedings, 7-17.

Pastor JV, Arrègle J, Palomares A (2001). Diesel spray image segmentation with a likelihood ratio test. Applied optics 40(17):2876-85.

Payri R, García JM, Salvador FJ, Gimeno J (2005). Using spray momentum to understand the influence of diesel nozzle geometry on spray characteristics. Fuel 84:551-61.

Petit C, Reckers W, Becker JM, Jourlin M (2005). Characterization of diesel spray images using an original shape processing methodology. Image Anal Stereol 24(2):95104.

Pun T (1981). Entropic thresholding: a new approach. Comput Vision Graph 16:210-39. 
Seneschal J, Ducottet C, Schon JP, Champoussin JC, Gucher P (2003). Automatic system for visualization and characterization of high pressure diesel sprays. PSFVIP4, June 3-5, Chamonix, France, 1-7.
Soteriou C, Andrews R, Smith M, Torres N, Sankhalpara S (2000). The flow patterns and sprays of variable orifice nozzle geometries for diesel injection. SAE 2000-010943, 1007-29. 